\title{
Análisis de la apropiación tecnológica en el adulto mayor. Más allá de la edad*
}

\author{
[Versión en Castellano] \\ Analysis of Technological Appropriation by Elderly People. Beyond Age \\ Análise da apropriação tecnológica em idosos. Além da idade
}

Recibido el 5 de marzo, 2019. Aceptado el 25 de octubre, 2019.

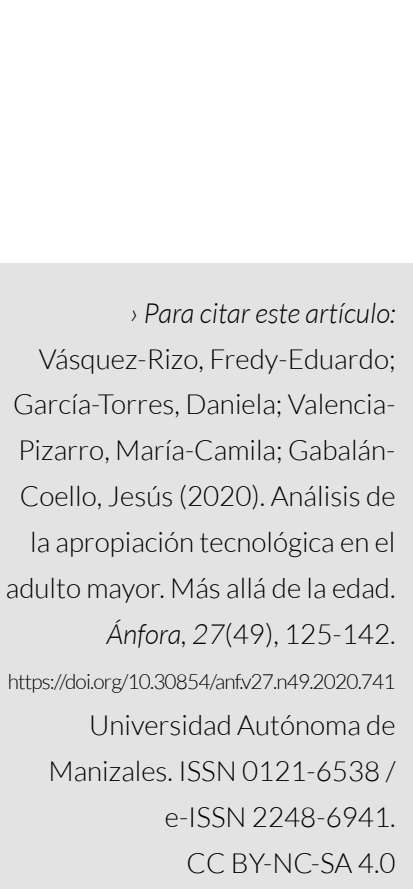

Fredy-Eduardo Vásquez-Rizo**

https://orcid.org/0000-0003-1398-6174

Colombia

Daniela García-Torres***

https://orcid.org/0000-0002-0791-4468

Colombia

María-Camila Valencia-Pizarro****

https://orcid.org/0000-0003-3450-4074

Colombia

Jesús Gabalán-Coello****

https://orcid.org/0000-0001-7674-8849

Colombia

* Este artículo se presenta como parte de los resultados finales de la investigación: "Análisis de la apropiación tecnológica en el adulto mayor", realizada en la Universidad Autónoma de Occidente, por los grupos de investigación en Educación y Gestión del Conocimiento y Sociedad de la Información. Los investigadores declaran que no hubo conflictos de intereses en la ejecución del proyecto de investigación.

** Ph.D en Gestión de la Información y de la Comunicación en las Organizaciones, Universidad de Murcia, España. Magíster en Ciencias de la Información y Administración del Conocimiento, Instituto Tecnológico de Estudios Superiores de Monterrey-ITESM, México. Docente Tiempo Completo, Universidad Autónoma de Occidente, Santiago de Cali, Colombia. Correo electrónico: fvasquez@uao.edu.co

*** Estudiante de Comunicación Social-Periodismo, Universidad Autónoma de Occidente, Santiago de Cali, Colombia.Correo electrónico: daniela.garcia_tor@uao.edu.co

**** Estudiante de Comunicación Social-Periodismo, Universidad Autónoma de Occidente, Santiago de Cali, Colombia. Correo electrónico: maria_c.valencia_p@uao.edu.co

***** Ph.D en Medición y Evaluación en Educación, Universidad de Montreal, Canadá. Magíster en Ingeniería con énfasis en Ingeniería Industrial, Universidad del Valle, Colombia. Vicerrector Académico, Universidad Católica de Pereira, Pereira, Colombia. Correo electrónico: jesus.gabalan@ucp.edu.co 


\title{
Resumen
}

Objetivos: evidenciar las condiciones actuales de acceso del adulto mayor a la era digital y sus reales opciones de apropiación tecnológica, en la ciudad de Santiago de Cali (Colombia). Metodología: se recurre a una técnica de análisis cualitativo aplicada a un grupo focal de adultos de la tercera edad, a través del cual se trabajan diferentes aspectos relacionados con sus comportamientos y necesidades asociadas a la era digital. Se realizaron entrevistas acerca de temas como interés tecnológico, responsabilidad estatal, alfabetización digital y apropiación tecnológica, buscando determinar su postura frente al tema y su posibilidad de inserción real. Resultados: se halló que el grupo focal asume que la tecnología es lo que mueve el mundo de hoy; los 10 participantes son conscientes de que es imprescindible el uso de internet para realizar muchas de las acciones cotidianas como informarse, comunicar, compartir, aprender y comercializar. Sin embargo, el 100\% de los participantes expresó que la tecnología está más enfocada a satisfacer las necesidades de los jóvenes que de los adultos mayores y quisieran estar a la par. También reconocen que su manejo de artefactos y aplicaciones es muy limitado, motivo que les hace sentirse excluidos. Conclusiones: se concluye que los adultos mayores constituyen una población en latente riesgo de exclusión tecnológica, debido a diversos factores de tipo personal, motivacional, formativo, social y gubernamental que dificultan su incorporación efectiva en la sociedad de la información.

Palabras-clave: Tecnología adecuada; Adulto y tecnología; Inserción tecnológica del adulto; Alfabetización digital; Apropiación digital.

\begin{abstract}
Objective: to demonstrate the current access conditions to the digital age by the elderly people and their true options of technological appropriation, in the city of Santiago de Cali (Colombia). Methodology: a qualitative analysis technique is applied to a focus group of elderly people to deal with different issues related to their behaviors and needs associated with the digital age. Interviews were conducted on topics such as technological interest, State responsibility, digital literacy and technological appropriation, seeking to determine their views on the issue and their possibility of real insertion. Results: findings show that the focus group assumes technology as the driver of today's world. The 10 participants are aware of the use of Internet as the key to perform many of the daily activities such as obtaining information, communication, sharing, learning and marketing. However, the 100\% of the participants expressed that the technology is more focused on meeting the
\end{abstract}


needs of young people than of the elderly, and they would like to keep up with it. They also recognize that their management of tools and applications is very limited, which makes them feel excluded. Conclusions: it is concluded that the elderly is a population at risk of technological exclusion due to a variety of factors such as personal, motivational, educational, social and governmental factors which hinder their effective incorporation into the information society.

Keywords: Appropriate technology; Adult and technology; Technological insertion of elderly. Digital literacy; Digital appropriation.

\section{Resumo}

Objetivo: demonstrar as condições atuais de acesso dos idosos à era digital e suas reais opções de apropriação tecnológica, na cidade de Santiago de Cali (Colômbia). Metodologia: utiliza uma técnica de análise qualitativa aplicada a um grupo focal de adultos idosos, através do qual são trabalhados diferentes aspectos relacionados a seus comportamentos e necessidades associadas à era digital. Foram realizadas entrevistas sobre temas como interesse tecnológico, responsabilidade estatal, alfabetização digital e apropriação tecnológica, buscando determinar sua posição sobre o assunto e sua possibilidade de inserção real. Resultados: verificouse que o grupo focal assume que a tecnologia é o que move o mundo hoje; Os 10 participantes estão cientes de que o uso da Internet é essencial para executar muitas das ações diárias, como informação, comunicação, compartilhamento, aprendizado e marketing. No entanto, 100\% dos participantes expressaram que a tecnologia está mais focada em atender às necessidades dos jovens do que dos adultos mais velhos e eles gostariam de estar a par. Eles também reconhecem que seu gerenciamento de artefatos e aplicativos é muito limitado, o que os faz sentir excluídos. Conclusões: conclui-se que os idosos constituem uma população em risco latente de exclusão tecnológica, devido a vários fatores pessoais, motivacionais, de treinamento, sociais e governamentais que dificultam sua efetiva incorporação na sociedade da informação.

Palavras-chave: Tecnologia apropriada; Adulto e tecnologia; Inserção tecnológica do adulto, Alfabetização digital; Apropriação digital. 


\section{Introducción}

Este artículo aborda una problemática que tiene como protagonista al adulto mayor, una persona que, según el Congreso de la República de Colombia (2008), tiene una edad de 60 años o más y que, por razones asociadas al avance tecnológico desmedido y apresurado de la sociedad de la información actual, no cuenta con las mismas condiciones de inclusión, dadas sus características y precarias opciones de alfabetización y apropiación digital.

Y es que cuando se habla de sociedad de la información, un término acuñado por autores contemporáneos (Carrillo-Gamboa y Batra, 2012; Mostafa y Sabbag, 2016; Cezar y Suaiden, 2017; Ziemba, 2019), se debe pensar en un gran nicho social donde cada individuo tenga las mismas posibilidades de desarrollo y participación, sin importar su edad, género o condición; una sociedad realmente democrática en la que todos los ciudadanos cuenten con los mismos derechos, que no excluya y que permita que los individuos disfruten de las maravillas de un mundo moderno (Usgame-Zubieta y Charum, 2005).

En este orden de ideas, una de esas maravillas es precisamente el acceso y uso de internet, herramienta que se constituye en el puente comunicante entre los individuos y aquel mundo moderno (Vásquez-Rizo y Gabalán-Coello, 2017); un mundo que tiene como ámbito de desarrollo a la sociedad de la información, la cual ofrece un cúmulo sin fin de alternativas de inclusión asociadas a la era digital. Sin embargo, tal contexto presenta latentes desafíos y retos, máxime para aquellas poblaciones como la de los adultos mayores, cuyo desarrollo, alfabetización e inclusión involucra diversos factores asociados a las características particulares de sus sujetos, entre los que se pueden mencionar aspectos como el desconocimiento de elementos de seguridad digital y privacidad o la ansiedad que puede suscitar el descubrimiento de lo nuevo o el exceso de horas de conexión, entre otros.

Es así como en un mundo cambiante, en permanente evolución, el acceso y conocimiento tecnológico se constituye en un elemento a favor o en contra de las personas (Schoemaker y Tetlock, 2017); y en esa cotidianidad de información y conocimiento el adulto mayor es uno de los individuos más olvidados y uno de los sujetos más vulnerables a las condiciones de velocidad y rápida obsolescencia de los artefactos que se requiere para vivir en este mundo moderno.

Por lo anterior, el presente estudio pretende evidenciar las condiciones actuales de acceso del adulto mayor a la era digital, contando como escenario con la ciudad de Santiago de Cali, Colombia, sus reales opciones de apropiación tecnológica y sus posibilidades de alfabetización. 


\section{El adulto mayor como sujeto de análisis}

Para empezar a soportar teóricamente este tema, se hace necesario entender y evaluar la posibilidad real que tiene el adulto mayor en la adquisición y uso de las Tecnologías de la Información y la Comunicación - TIC, en especial desde el punto de vista de la educación y la formación permanente, inquietud respaldada por las posturas de Dogruel, Joeckel y Bowman (2015) y Zilidis y Zilidou (2018).

Según Merchán-Maroto y Cifuentes-Cáceres (2014), citando el aporte a la Teoría de la Desvinculación de Cumming y Henry (1961), el adulto mayor es un sujeto que actúa, de manera general, de acuerdo con lo que le propone su voluntad o lo que le produce motivación o interés; razón por esta razón, al ser sus obligaciones reducidas, asume la mayoría de sus actividades en aislamiento, desvinculándose o siendo desvinculado, en parte, por el contexto social, pero no porque él así lo quiera sino porque los otros así lo conciben. Tal posición es compartida por Motte y Moñoz (2002), al afirmar que el adulto mayor desarrolla una imagen positiva de sí mismo sí y solo sí se siente vinculado a algo útil y continuo, lastimosamente éste no es el pensar de los demás miembros de la sociedad.

Es por esto que, según Mogollón-González (2014), entre más incluido se sienta el adulto mayor en el uso de las TIC, a través del acceso a internet y demás equipamientos, mejor será su avance cognitivo respecto al mundo digital; pero esto debe ser claro no solo para el adulto sino para su comunidad.

Es así como debe aparecer un elemento crucial para trascender en esta claridad y que el adulto mayor se sienta incluido en el tema y adquiera "sensibilidad" al respecto, y es aquí donde justamente la educación entra a ser un aspecto fundamental, pues es la educación el proceso a través del cual el adulto mayor disminuye los riesgos de diezmar su capacidad cognitiva, incrementando sus opciones de inserción social, y contribuyendo de esta manera a su bienestar personal (Centro Latinoamericano y Caribeño de Demografía [CELADE] y Comisión Económica para América Latina y el Caribe [CEPAL], 2011).

\section{Lo tecnológico como instrumento de inclusión (o exclusión)}

Pero antes de hablar de manera concreta de la educación en el adulto mayor, es importante brindar claridad acerca del concepto de tecnología, entendida ésta, para efectos del presente artículo, según San Martín y Guisen (2016), como aquel mecanismo que debe responder a un enfoque socio-técnico que posibilite la inclusión social de quien lo utiliza, en un determinado contexto de trabajo colaborativo, siendo parte de una actividad conjunta (pensamiento sistémico) así la participación del sujeto en dicho conglomerado no sea significativa o evidente. 
Es decir, una tecnología que sirva como herramienta para generar dinámicas menos excluyentes, potenciadoras de las capacidades de los individuos y sus contextos y posibilitadoras de comunicaciones efectivas, que permitan ejercer y gozar de los derechos individuales y colectivos, en función de verdaderas Prácticas Educativas Mediatizadas -PEM (Marín-Ossa, 2015).

Para nadie es un secreto que las TIC han introducido de manera progresiva y significativa cambios trascendentales en los procesos educativos, productivos y empresariales, en las formas de trabajo y en la vida cotidiana de las personas (Carnoy, 2002). Esta postura es compartida por Kaschig, Maier y Sandow (2016), quienes reconocen que la implementación tecnológica ha dado lugar a nuevas posibilidades y estructuras de trabajo colaborativo, permitiendo una total interrelación entre las personas, los conjuntos de personas y los entornos que éstas comparten.

Y es justamente esto lo que una sociedad responsable debería permitir con la incorporación digital y tecnológica del adulto mayor en verdaderos sistemas articulados de desarrollo, que le otorguen el espacio para realizar acciones en interacción responsable y en igualdad de condiciones en contextos educativos agradecidos, preocupados, transformadores e incluyentes.

\section{Un cambio de paradigma desde la educación}

La llegada de las TIC y la aparición y desarrollo de una sociedad de la información dispuesta en red ha obligado a tener un conocimiento que posibilite su uso e inserción efectiva. Es por esto que la sociedad de hoy requiere nuevas competencias, capacidades y habilidades, todas ellas asociadas al mundo digital. Lastimosamente, dicha adquisición no es posible con la formación tradicional, razón por la cual necesita la ayuda de la educación continua. Al respecto, Silvera (2005) habla de esto más que como una necesidad, como un derecho inherente al desarrollo de las personas que viven en una sociedad digitalizada y moderna. Esto, porque no se puede concebir que la educación siga siendo un proceso dependiente de los materiales tangibles, sino que se debe ver desde una perspectiva más profunda y es a través de la educación permanente en TIC que se puede llegar a una real "alfabetización en destrezas” (Irrazabal-Paz y Loutayf, 2014).

Dicha alfabetización, tendiente hacia la apropiación, no busca otra cosa que lograr en el individuo (en este caso el adulto mayor), la acreditación de aquellas destrezas y prácticas en TIC necesarias para su desenvolvimiento en la era digital, es decir, en la vida misma, sin que tenga que ser resignado su historial de conocimiento; al contrario, lo que se busca con dicha forma de educación es que incorpore a dicho bagaje un uso tecnológico (Chiu y Liu, 2017). 
De lo contrario, seguirá existiendo una polarización entre aquellos que se encuentran actualizados en materia de tecnología y aquellos que no lo están, abriendo aún más lo que algunos autores como Rodríguez-Gallardo (2012) y Molina-Durán (2017) denominan "brecha digital", que no es otra cosa que una brecha social y todo lo que este macro-concepto conlleva, donde el adulto mayor, como es innegable, se reconoce hoy como el máximo perjudicado de la evolución digital humana (CEPAL, 2008; Sunkel y Ullman, 2019).

\section{La educación tecnológica en el adulto mayor}

Para terminar este apartado teórico, se debe decir que según el informe de "Perspectivas de la Población Mundial”, elaborado por Naciones Unidas (UN, 2017), en la actualidad hay en el mundo más personas de edad avanzada por habitante que nunca antes, y esta proporción sigue en aumento.

Dicha situación ya había sido expuesta por la Organización de las Naciones Unidas para la Educación, la Ciencia y la Cultura, Unesco (1997), a través de la "Declaración de Hamburgo sobre la Educación de Adultos", en la que se hizo énfasis en la importancia de la no exclusión de estas personas en la sociedad, incluso con los advenimientos tecnológicos, permitiéndoles la posibilidad de aprender en igualdad de condiciones y de maneras apropiadas, reconociendo, valorando y utilizando sus capacidades, competencias y potencialidades.

Lastimosamente, esto en la práctica no está ocurriendo, pues pareciera que la mayoría de los programas en pro de una alfabetización digital están siendo dirigidos a mejorar las competencias de los jóvenes durante sus distintos niveles de formación (educación primaria, secundaria y superior), ignorando en sus planes de estudio y capacitación a las personas en edad avanzada, situación que se puede constatar en los pensum de los programas y prerrequisitos y requisitos de los cursos existentes en la actualidad.

Y es aquí donde justamente este artículo quiere incidir, haciendo un llamado de atención a la sociedad para que se entienda que, si bien el adulto mayor necesita una ayuda adicional o distinta para su inclusión efectiva en la sociedad de la información, se debe comprender que su proceso de formación debe ser paciente y permanente, que debe iniciar por los aspectos más básicos, involucrando los actos más simples y quizás hasta “obvios” (para el resto) en este campo.

Es por esto que dicha educación, que más que especializada debe ser focalizada a unas necesidades fundamentales; debe caracterizarse por brindar al adulto mayor un entorno rico en estímulos, incorporando, según Agudo, Pascual y Fombona (2012), nuevos modelos y estrategias de acción. De esta forma, el adulto mayor podrá aprender con las TIC, involucrando el diseño y creación per- 
manente de nuevos espacios de aprendizaje y comunicación, capaces de gestionar la demanda existente por parte de este importante conjunto de personas.

Como bien se ha mencionado, este ejercicio se desarrolla en la ciudad de Santiago de Cali, una urbe que intenta paulatinamente adentrarse al desarrollo digital, donde, como en el resto del mundo, muchas de las actividades actuales giran alrededor de internet y la tecnología.

Dicha realidad citadina hace que todos sus individuos, incluidos los adultos mayores, quieran formar parte de este escenario; sin embargo, existen aún ciertos elementos que no permiten que dicho ámbito trascienda, entre los que se cuentan, entre otros, las insuficientes campañas de alfabetización digital, el desinterés del Gobierno y las instituciones de educación y la desidia de algunas personas por incluir o ayudar a incorporar al adulto mayor en esta dinámica (incluso, en ocasiones, por parte de estos mismos sujetos de edad avanzada).

\section{Metodología}

En esta investigación se optó por aplicar la técnica de análisis cualitativo, a través de un grupo focal, realizado entre el 9 de febrero y el 24 de mayo de 2018, conformado por 10 personas (cinco hombres, cinco mujeres), con edades superiores a los 60 años, de estratos 2 a 3 y con un nivel de formación básico (entre primaria y bachillerato). Esto con el fin de entender el comportamiento, las falencias, los atributos y las necesidades que tienen los adultos mayores en cuanto a su posibilidad de inclusión efectiva en el mundo tecnológico y digital.

De esta manera, se intentó identificar la interacción y adaptación que tienen los adultos mayores con las tecnológicas y su facilidad de manejo, así como determinar el nivel de consciencia que el grupo focal tiene acerca de la importancia de su uso e implementación.

Para ello, se plantean preguntas relacionadas con los siguientes aspectos: interés en la tecnología, responsabilidad estatal en el tema, alfabetización digital, apropiación tecnológica, factores humanos que inciden en el uso de la tecnología, posibilidades de formación y capacitación, acceso y uso, conocimiento tecnológico, entre otros.

Dichos aspectos permiten esbozar un panorama general asociado a esta temática, con la intención de sugerir posibles alternativas para la ciudadanía mayor de la ciudad de Santiago de Cali, así como detectar elementos positivos y negativos vinculados a dicho proceso de inclusión social moderna. 


\section{Resultados}

Se pudo establecer que para el grupo focal seleccionado es una realidad inapelable que la tecnología "es lo que mueve el mundo", por lo que afirman que "son conscientes que es imprescindible el uso de la red (internet) para realizar cualquier tipo de acción, incluso las más cotidianas, como pueden ser: informarse, comunicarse, compartir, aprender y comercializar". En el mismo sentido, un adulto mayor manifiesta:

"Los mecanismos antiguos para comunicarnos o informarnos se están quedando obsoletos, en el pasado, es hora de adaptarnos a este nuevo sistema, que siendo bien utilizado podría darnos resultados sorprendentes e inimaginables... todos aquí debemos entender que estamos en un nuevo siglo y que el futuro nos está haciendo un llamado".

Sin embargo, el 100\% de los participantes reconoce que dicha tecnología está más enfocada a satisfacer las necesidades de los jóvenes que de los adultos mayores, pero sí quisieran acceder a ella y estar a la par de dichos jóvenes. También reconocen que su conocimiento en el manejo y en todo lo que la tecnología presenta es muy limitado, motivo por el cual tienden a sentirse excluidos de esta sociedad de la información.

Por otro lado, que para ellos la educación (sin detenerse en especificidades o conocimientos avanzados en el tema) debe ser la manera en que pueden llegar a apropiarse medianamente de las TIC; y si bien entienden que no podrían arrancar desde cero debido a su edad, sí podrían someterse a algún tipo de programa que les permitiera introducirse en la temática o acercarse a este nuevo conocimiento. Para la mayoría de los adultos mayores encuestados, las actuales generaciones tienen la ventaja que desde muy pequeños se vinculan, a través de la educación, en esta nueva dinámica, así esta educación digital aún no sea perfecta; empero, manifiestan su temor acerca de los peligros que para niños y jóvenes de hoy representa exponerse a las TIC, en especial, las redes sociales, debido a que opinan que son un escenario demasiado permisivo.

Ahora, cuando a los adultos mayores se les indaga acerca del papel del gobierno en esta alfabetización digital para su generación, sostienen que no se sienten incluidos en los planes del Estado. Afirman que desde la misma concepción de las políticas educativas estatales (Ministerio de Tecnologías de la Información y Comunicaciones, 2018), se evidencia que no existe una intención real de articularlos a este contexto, soportando su opinión principalmente en el imaginario social que los desacredita como sujetos competentes para manipular dichos artefactos. Aquí plantean el siguiente interrogante: “¿cómo quieren que 
seamos competentes y que nos articulemos a la sociedad de la información si no nos capacitan para ello?”.

En relación con lo anterior, un adulto mayor expresa: "el otro día supe de un taller que realizaba la Alcaldía en manejo de tecnología... era algo básico, supongo... pero no siempre sucede...”.

Al escuchar este aporte una adulta mayor agrega: “...eso es cierto, también supe de ese taller y me inscribí, porque no quiero seguir estando rezagada en algo que mis nietos ya me llevan ventaja, pero cuando fue a iniciar el curso la sorpresa fue que solo estábamos inscritas cuatro personas, por lo que el taller nunca se realizó y luego ya no supe más...”.

Con este testimonio se pone en evidencia una problemática adicional a las que ya se han abordado y es el desinterés de muchos adultos mayores por estos temas. Si bien el gobierno peca en algunos aspectos, el ciudadano mayor también omite las pocas opciones estatales o institucionales que se le brindan (Paz-Saavedra, 2008); es por esto que, desde el punto de vista económico, muchas veces no es viable o rentable generar programas o cursos sobre tecnología, enfocados de forma exclusiva en este tipo de públicos.

A lo anterior, se suma el temor que puede sentir el adulto mayor por lo nuevo o por aquello que le es ajeno, incluso el miedo al error, la burla o el compromiso que se asume con una nueva responsabilidad, para la cual aún no se siente completamente preparado o que no tiene el control, pues en su gran mayoría prefieren mantener la comodidad de lo ya aprendido y no quieren experimentar con nuevas actividades, en especial si van más allá de la dimensión tradicional de los sentidos (Pérez-Díaz, 2009).

Por otro lado, también comentan que la familia, los amigos, los vecinos y la sociedad que los rodea tampoco tienen mayor intención (o paciencia) en involucrarlos en estos temas; algunos expresaron que muchos de sus allegados consideran una pérdida de tiempo llevar los adultos mayores al manejo de los recursos tecnológicos y, de esta manera, juzgan incluso sus capacidades motoras e intelectuales, aduciendo que no les permiten habilidades suficientes para intentar siquiera adquirir este tipo de saberes.

Uno de los entrevistados precisa:

"la sociedad actual no puede vanagloriarse de convivir en un mundo globalizado si no tiene en cuenta a todas las personas, bien sea por edad, como es nuestro caso, o por otros factores (poder adquisitivo, condición social, género...), pues es evidente que solo algunos sectores, en especial los ricos y poderosos, son quienes gozan de todos los privilegios y los derechos a la información...”. 
Y otro se suma diciendo que:

"la sociedad debe estar preparada para expandir las tecnologías e internet a todas las personas, teniendo en cuenta cada condición particular o situación específica....... sé que es algo difícil, pero pues ya lo sufrimos nosotros, solo espero que esto no ocurra con los jóvenes de ahora cuando lleguen a viejos y la tecnología que conozcan se vuelva obsoleta”.

Todas estas opiniones, si bien pueden sonar como excusas para justificar la falta de interés del adulto mayor en el tema o para soportar su exclusión de los actuales escenarios por parte de la sociedad, no deja de ser preocupante cuando existe un interés generalizado, por lo menos desde la academia, por disminuir la brecha digital y tecnológica existente, en la cual este grupo poblacional es uno de los más afectados.

Así pues, en materia digital, ser adulto mayor no debe ser una sentencia de muerte, sino que debe entenderse como una etapa más de la vida en la que se puede seguir creciendo intelectualmente (Fernández, Penecino y Ascolani, 2016); una fase de la existencia en la cual la enseñanza en el manejo de las TIC debe ser un estímulo permanente, un aliciente, incluso, para disminuir los riesgos de algunas enfermedades cognoscitivas o de algunos estados clínicos depresivos (Américas 5G, 2018).

Es por esto que, entre los testimonios que recopiló este estudio también está la voz de quienes reclaman inclusión en los procesos formales y, a la vez, manifiestan que en parte la decisión de aprender es personal: "Los adultos mayores debemos sentirnos parte de esta sociedad, no nos podemos aislar o dejarnos aislar pensando en argumentos inválidos. Somos seres diseñados para aprender y para razonar, lo cual nos permite adquirir conocimientos sin importar la etapa de la vida en la que estemos".

En síntesis, gracias a la participación de un grupo focal compuesto por 10 personas, pobladores de la ciudad de Santiago de Cali, se pudo establecer que desde el punto de vista personal, el adulto mayor muchas veces carece de motivaciones que le permitan interesarse en formar parte de dicha sociedad o de aprender siquiera los aspectos básicos del manejo de la tecnología. Situación que se acrecienta cuando éste se siente vulnerado o impedido en el ejercicio de sus derechos como ciudadano o cuando entiende que nadie se interesa porque participe en contextos asociados a la era digital. 


\section{Conclusiones}

Los adultos mayores constituyen una población en latente riesgo de exclusión tecnológica. Esto se debe a que existen factores de tipo personal, formativo, social y gubernamental que dificultan su inserción efectiva en la sociedad de la información.

Desde el punto de vista de lo formativo, si bien la educación tecnológica para el adulto mayor existe, ésta ha fallado en su real preocupación porque éstos tengan una mayor participación e interacción, tanto con los diferentes actores sociales como con la misma tecnología; por tal razón, se necesitan más y mejores campañas de alfabetización y apropiación digital para esta población o una más amplia atención de parte de las instituciones de educación para que generen programas y cursos tendientes a fomentar y fortalecer este tipo de capacitación.

Dicha capacitación también debe dárseles a los demás en especial a aquellos cercanos a la población objeto de estudio, dado que, desde el punto de vista social, de nada sirve que los adultos mayores se capaciten en dichas habilidades y competencias si quienes les rodean no están atentas o dispuestas a incorporarlos a sus actividades digitales; situación que aplica para todos los estamentos sociales.

Por todo lo anterior, es menester de los entes gubernamentales sumarse a esta iniciativa, debido a que son los que establecen las leyes y normativas que pueden hacer viable esta situación "ideal" de inclusión, uso y acceso para el adulto mayor; además, es el gobierno el responsable de brindar las condiciones suficientes para que estas personas se sientan insertas en la sociedad de la información, aceptadas y respaldadas por sus conciudadanos, amparadas por el sistema educativo y motivadas de manera individual y colectiva, en procura de un real involucramiento en la era tecnológica, tendiente a homogenizar un poco la ya profunda brecha digital.

Por último, se debe decir que todo lo aquí descrito da pie para que desde la academia, por lo menos desde la Institución de Educación Superior -IES- se asuma un compromiso para con la comunidad, convirtiendo esta temática en un asunto de interés no solo para un ejercicio investigativo sino para que sea un elemento constitutivo de sus políticas de responsabilidad social; ello, implica que la Universidad debe desarrollar alternativas de capacitación no tradicional (ausentes actualmente en el marco de sus programas), teniendo como sujetos de alfabetización digital a esta población vulnerable, así como a otras distantes del ideal de la sociedad de información.

De esta forma se logrará la comunión entre el sistema educativo convencional (respaldado por políticas que orientan su labor desde el gobierno) y los conglomerados sociales que requieren de sus servicios, siendo la adultez mayor uno de ellos. 
Por eso, se recomienda atender lo aquí expuesto, con el fin de intentar reducir la brecha digital, por lo menos a pequeña escala, para así ir permeando escenarios más amplios, con la intención de promover el empoderamiento de los adultos mayores sobre el manejo de las TIC y de todos los elementos asociados al mundo digital.

\section{Referencias}

Agudo, S.; Pascual, M.; Fombona, J. (2012). Uso de las herramientas digitales entre las personas mayores. Comunicar. Revista Científica de Comunicación y Educación, 39(20), 193-201. Grupo Comunicar. https://doi.org/10.3916/ C39-2012-03-10 [Consultado el 13 de febrero de 2019].

Américas 5G (2018). TIC para adultos mayores en América Latina. Bellevue, WA: 5G Américas. https://www.crcom.gov.co/recursos_user/2018/ac_reg/cond_ etm/coment/5G-AMERICAS.pdf [Consultado el 13 de febrero de 2019].

Carnoy, M. (2002). Sustaining the New Economy. Work, Family, and Community in the Information Age. Boston, MA: Harvard University Press.

Carrillo-Gamboa, F. J.; Batra, S. (2012). Understanding and Measurement: Perspectives on the Evolution of Knowledge-based Development. International Journal of Knowledge-Based Development, 3(1), 1-16. Inderscience Publishers. https://doi.org/10.1504/IJKBD.2012.045568 [Consultado el 13 de febrero de 2019].

Centro Latinoamericano y Caribeño de Demografía-CELADE y Comisión Económica para América Latina y el Caribe-CEPAL. (2011). Los derechos de las personas mayores. Materiales de estudio y divulgación. Hacia un cambio de paradigma sobre el envejecimiento y la vejez.

https://repositorio.cepal.org/handle/11362/21497

[Consultado el 13 de febrero de 2019].

Cezar, K. G.; Suaiden, E. J. (2017). O impacto da sociedade da informação no processo de desenvolvimento. Informacao E Sociedade: Estudos, 27(3), 19-29. Universidade Federal Da Paraíba.

https://periodicos.ufpb.br/index.php/ies/article/view/34305/18967 [Consultado el 10 de octubre de 2018]. 
Chiu, C. J.; Liu, C. W. (2017). Understanding Older Adult's Technology Adoption and Withdrawal for Elderly Care and Education: Mixed Method Analysis from National Survey. Journal of Medical Internet Research, 19(11), 49-49. National Institutes of Health. https://www.ncbi.nlm.nih. gov/pubmed/29101093 [Consultado el 12 de octubre de 2019].

Comisión Económica para América Latina y el Caribe, CEPAL. (2008). La sociedad de la información en América Latina y el Caribe: desarrollo de las tecnologías y tecnologías para el desarrollo. https://repositorio.cepal.org/handle/11362/2537 [Consultado el 10 de octubre de 2018].

Congreso de la República de Colombia. (2008). Ley 1251 de 2008.

https://www.icbf.gov.co/cargues/avance/docs/ley_1251_2008.htm [Consultado el 8 de febrero de 2019].

Cumming, E.; Henry, W. E. (1961). Growing Old: The Process of Disengagement. New York, NY: Basic Books.

Dogruel, L.; Joeckel, S.; Bowman, N. D. (2015). The Use and Acceptance of New Media Entertainment Technology by Elderly Users: Development of an Expanded Technology Acceptance Model. Behaviour \& Information Technology, 34(11), 1052-1063. https://www.tandfonline.com/doi/ $\mathrm{pdf} / 10.1080 / 0144929 X .2015 .1077890$ ? needAccess $=$ true [Consultado el 11 de octubre de 2018].

Fernández, F. M.; Penecino, E.; Ascolani, D. (2016). Educación tecnológica en adultos mayores. En VIII Congreso Internacional de Investigación y Práctica Profesional en Psicología - XXIII Jornadas de Investigación - XII Encuentro de Investigadores en Psicología del MERCOSUR, Buenos Aires, Argentina. https://www.aacademica.org/000-044/402.pdf

[Consultado el 8 de febrero de 2019].

Irrazabal-Paz, M. F.; Loutayf, M. S. (2014). La “alfabetización” en el siglo XXI: el desarrollo de habilidades digitales en la enseñanza de lectura y escritura. En Actas Congreso Nacional Subsede Cátedra Unesco UNR, octubre, Rosario, Argentina. https://rephip.unr.edu.ar/bitstream/handle/2133/4835/ Irrazabal\%20Paz\%20-\%20Loutayf.pdf? sequence $=3 \&$ isAllowed $=y$ [Consultado el 17 de febrero de 2019].

Kaschig, A.; Maier, R.; Sandow, A. (2016). The Effects of Collecting and Connecting Activities on Knowledge Creation in Organizations. Journal of 
Strategic Information Systems, 25(4), 243-258. https://doi.org/10.1016/j. jsis.2016.08.002 [Consultado el 13 de febrero de 2019].

Marín-Ossa, D. L. (2015). La educación mediatizada. Distancias y aproximaciones conceptuales en las metodologías de mediatización del conocimiento. Revista da FAEEBA - Educação e Contemporaneidade, 24(44), 41-53. https://www.revistas.uneb.br/index.php/faeeba/article/view/1819/1233 [Consultado el 12 de febrero de 2019].

Merchán-Maroto, E.; Cifuentes-Cáceres, R. (2014). Tema 6. Teorías psicosociales del envejecimiento. http://asociacionciceron.org/wp-content/ uploads/2014/03/000001 17-teorias-psicosociales-del-envejecimiento.pdf [Consultado el 8 de febrero de 2019].

Ministerio de Tecnologías de la Información y Comunicaciones, MinTIC. (2018). Alfabetización digital. http://www.mintic.gov.co/portal/604/w3-article-5447.html [Consultado el 2 de marzo de 2019].

Mogollón-González, E. J. (2014). Una propuesta para el mejoramiento cognitivo en el adulto mayor: una alternativa al entrenamiento cerebral. $R \boldsymbol{e}$ vista Electrónica Educare, 18(2), 1-17. Universidad Nacional de Costa Rica. https://www.revistas.una.ac.cr/index.php/EDUCARE/article/ view/5830/16192 [Consultado el 14 de febrero de 2019].

Molina-Durán, J. V. (2017). Las implicaciones de la brecha digital para los países en desarrollo: caso Venezuela. Sapienza Organizacional, 5(9), 105-128. http://erevistas.saber.ula.ve/index.php/sapienza/article/view/9976/9905 [Consultado el 1 de marzo de 2019].

Mostafa, S. P.; Sabbag, D. (2016). A relação saber-poder na organização e repre-sentaçao do conhecimento. Scire, 22(1), 15-24.

https://www.ibersid.eu/ojs/index.php/scire/article/view/4296/3828 [Consultado el 28 de febrero de 2019].

Motte, A.; Moñoz, J. (2002). Envelhecimento social. En J. Muñoz (coord.). Psicología del envejecimiento (pp. 95-110). Madrid, España: Pirámide.

Naciones Unidas, UN. (2017). World Population Prospects. The 2017 Revision. Key Findings and Advance Tables. https://esa.un.org/unpd/wpp/Publications/ Files/WPP2017_KeyFindings.pdf [Consultado el 26 de febrero de 2019]. 
Organización de las Naciones Unidas para la Educación, la Ciencia y la Cultura, Unesco. (1997). Declaración de Hamburgo sobre la Educación de Adultos. "El saber adulto, una clave para el siglo XXI”. http:/ / www.fundacion.uocra.org/ documentos/recursos/declaraciones/LA-DECLARACION-DE-HAMBURGO.pdf [Consultado el 8 de febrero de 2019].

Paz-Saavedra, L. E. (2008). Alfabetización digital en el adulto maduro una estrategia para la inclusión social. E-mail Educativo, 1, 1-16. Universidad Nacional de Colombia.

https://revistas.unal.edu.co/index.php/email/article/view/12486/13224 [Consultado el 28 de febrero de 2019].

Pérez-Díaz, R. (2009). Trastornos de ansiedad fóbica en el adulto mayor. Valoración crítica y manejo terapéutico. Geroinfo. Publicación de Gerontología y Geriatría, 4(1), 1-22. Centro de Investigaciones sobre: Longevidad, Envejecimiento y Juventud-CITED. https://docplayer.es/15869384-Geroinfo-publicacion-de-gerontologia-y-geriatria-tratamiento-de-la-ansiedad-en-el-adulto-mayor-resumen.html [Consultado el 28 de febrero de 2019].

Rodríguez-Gallardo, A. (2012). Tecnologías de la información y brecha digital en México 2001-2005. México D. F.: Universidad Nacional Autónoma de México-UNAM.

San Martín, P. S.; Guisen, M. A. (2016). Hacia las tecnologías para la inclusión social en contextos educativos regionales: análisis del caso "ECCA". Revista Electrónica "Actualidades Investigativas en Educación", 16(2), 1-26. Universidad de Costa Rica. https://revistas.ucr.ac.cr/index.php/aie/article/ view/23564/23785 [Consultado el 11 de febrero de 2019].

Schoemaker, J. H.; Tetlock, P. E. (2017). Building a More Intelligent Enterprise. MIT Sloan Management Review, 58(3), 28-38. Massachusetts Institute of Technology-MIT. https://sloanreview.mit.edu/article/building-a-more-intelligent-enterprise/ [Consultado el 26 de febrero de 2019].

Silvera, C. (2005). La alfabetización digital: una herramienta para alcanzar el desarrollo y la equidad en los países de América latina y el Caribe. Acimed, 1(13), 1-8. Centro Nacional de Información sobre Ciencias Médicas en Cuba. http://scielo.sld.cu/pdf/aci/v13n1/aci04105.pdf

[Consultado el 28 de febrero de 2019]. 
Sunkel, G.; Ullman, H. (2019). Las personas mayores de América Latina en la era digital: superación de la brecha digital. Revista de la CEPAL, 127, 243-268. Comisión Económica para América Latina y el Caribe -CEPAL. https://repositorio.cepal.org/bitstream/handle/11362/44580/1/ RVE127_Sunkel.pdf [Consultado el 13 de octubre de 2019].

Usgame-Zubieta, D.; Charum, J. (2005). Redes tecnoeconómicas entre productores y usuarios de conocimiento. En Red Iberoamericana e Interamericana de Indicadores de Ciencia y Tecnología-RICYT (org.). Indicadores de ciencia y tecnología en Iberoamérica. Agenda 2005 (pp. 156-192). Bogotá: Observatorio Colombiano de Ciencia y Tecnología-OcyT.

Vásquez-Rizo, F. E.; Gabalán-Coello, J. (2017). Agregando valor a las IES a través de la búsqueda y selección de información. Prisma Social. Revista de Ciencias Sociales, $18(1)$, 592-602.

https://revistaprismasocial.es/article/view/1427/1679

[Consultado el 28 de febrero de 2019].

Ziemba, E. (2019). The contribution of ICT Adoption to the Sustainable Information Society. Journal of Computer Information Systems, 59(2), 116-126. https://www.tandfonline.com/doi/ pdf/10.1080/08874417.2017.1312635? needAccess =true [Consultado el 10 de octubre de 2019 ].

Zilidis, G.; Zilidou, V. (2018). The Use of New Technologies Addressing Social Exclusion and Improving the Quality of Life of the Elderly. Interscientific Health Care, 1O(4), 10-16. University of Thessaly. http://www. inhealthcare.gr/manuscript/i-chrisi-ton-neon-technologion-pliroforias-kai-epikoinonias-stin-antimetopisi-toy-koinonikoy-apokleismoy-kai-tis-beltiosis-tis-poiotitas-zois-ton-ilikiomenon

[Consultado el 11 de octubre de 2018]. 
